\title{
Un audit des autorités fédérales américaines sur la brevetabilité du génome
}

\author{
Axel Kahn
}

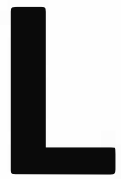

e 21 mai 1992, s'est tenu à Washington DC, dans le grand auditorium de l'Académie des Sciences des États-Unis, un audit sur la brevetabilité des séquences d'ADN. Les lecteurs de $\mathrm{m} / \mathrm{s}$ se rappelle que le NIH (National Institute of Health), en la personne de Craig Venter, a déposé fin 1991, puis début 1992, plus de 2500 séquences partielles d'ADNc devant le Bureau Américain des Brevets afin que lui soit reconnue la propriété intellectuelle de ces séquences $\left(m / s n^{\circ} 9\right.$, vol. 7, p. 960). Cette demande a entraîné aux ÉtatsUnis, en France et dans d'autres pays une émotion considérable. Dans notre pays, Monsieur Hubert Curien, ministre de la Recherche et de l'Espace, faisait connaître son opposition à une telle extension du droit des brevets, dans une lettre publiée par la revue Science $\left(n^{\circ} 5039\right.$, vol. 254, p. 1710). Le Comité National Consultatif d'Éthique (m/s $n^{\circ} 10$, vol. 7, p. 1114), l'Académie des Sciences et de nombreux scientifiques à titre personnel protestaient également contre la décision du NIH.

La réunion de Washington était organisée à la demande d'Alan Bromley, conseiller du président pour les affaires scientifiques, par un groupe de travail, le Genome Patent Working Group, composé de représentants du NIH (Bernardine Healy), du département d'État, de la NSF (National Science Fondation) (Mary E. Clutter), du DOE (Department of Energy), David J. Galas) et de l'Office of Science and Technology Policy (Donald A. Henderson). Les débats étaient présidés par Mary Clutter, de la NSF.

$m / s n^{\circ} 6$, vol. 8 , juin-juillet 92
La première intervention fut celle de Daniel Nathans, généticien humain et prix Nobel de Médecine qui, après un rappel des principes et objectifs de la recherche sur le génome, établit que les séquences partielles d'ADN complémentaire ne contenaient aucune information, et par conséquent ne lui semblaient pas pouvoir être brevetables. Robert Merges, professeur de Droit à l'Université de Boston, remarqua ensuite que, selon lui, certaines des revendications de la demande de brevet déposée par Craig Venter étaient irrecevables (par exemple ce qui touchait l'utilisation thérapeutique de ces séquences) alors que, en revanche, l'utilisation comme sonde pour isoler des gènes lui semblait parfaitement acceptable. L'orateur insistait néanmoins sur le caractère inextricable d'un système où un même gène serait couvert par des brevets différents, accordés pour des séquences partielles différentes déterminées dans divers laboratoires.

Vinrent ensuite les exposés des responsables des principales agences fédérales. La directrice du NIH, Berdardine Healy, exposa les arguments du NIH dont les éléments centraux, déjà connus, sont : le NIH a comme mission de favoriser le transfert et n'avait pas d'autre possibilité, en l'absence de règles en ce domaine, que de déposer une demande de brevet. Les molécules d'ADN complémentaire correspondant à des séquences partielles de gènes exprimés ne sont pas des molécules naturelles et sont, par conséquent, des réactifs parfaitement brevetables, notamment utiles comme sondes pour isoler des gènes. La politique du brevet permet, à la fois, de diffuser très rapidement l'information et de préserver toutes les chances d'une utilisation positive des données pour l'industrie américaine. A noter qu'au cours de son intervention, Bernardine Healy attaqua par deux fois la position française, en opposant le contenu de la lettre du ministre à Science et la prise de brevets sur des gènes humains par des équipes françaises, et en rejetant les références éthiques contenues dans cette lettre.

Le responsable du DOE, David Galas, indiqua que les séquences partielles d'ADN complémentaire ne correspondaient qu'à un stade tout à fait intermédiaire d'une recherche et ne contenaient pas, en elles-mêmes, d'informations dont l'utilité pouvait être décrite. De plus, le programme Génome Humain et les autres programmes Génome étant largement internationaux, la brevetabilité de ce type de données risquait d'être un obstacle à la circulation des résultats et, d'ailleurs, avait commencé de créer de difficiles problèmes dans la fluidité nécessaire de l'information et dans l'échange des réactifs entre les différents laboratoires du monde. David Galas remarquait également que les séquences partielles d'ADNc n'étaient que la partie émergée de l'iceberg, le même type de problème pouvant se poser pour n'importe quelle séquence d'ADN, même non codante, des cartes génomiques, des librairies d'ADN (cosmides, YAC, etc.) ou des microsatellites, ce qui pourrait, si on généralisait l'attitude préconisée par le NIH, constituer un frein considérable au développement de la recherche, et par ailleurs, vider 
de toute substance les brevets authentiques. Enfin, la représentante du Department of Commerce, Madame Deborah Wince-Smith, indiqua que les autorités fédérales amćricaines ćtaient absolument favorables à une protection plus forte de toutes les avancécs issues des laboratoires américains et que les agences fédérales aussi bien que les universités étaient fortement encouragées à développer cettc politique. Elle indiqua que $40 \%$ des nouveaux produits utilisćs en thérapcutique humainc étaient issus de recherches universitaires, soulignant ainsi l'importance considérable de l'implication des centres de recherche académiques dans les opérations de transfert.

Vinrent ensuite les interventions publiques, sous forme de prises de position brèves de cinq minutes. Parmi ces interventions, du côté américain, il faut retenir celle du Président de l'American Society of Human Genetics, Walter Nance, qui rappela son opposition absoluc à la brevetabilité de séquences tronquécs du type EST (Expressed Sequences Tag) et STS (Sequence Tagged Sites). Le représentant de IBA (Industrial Biotechnology Association), Richard Godown, indiqua que son association, représentant les plus grandes compagnies de biotechnologie américaines, fera connaître sa position officielle le 10 juin*. Néanmoins, il rapporta que de nombreuscs réserves avaient été émises sur la brevetabilité de séquences partielles d'ADN, considérant qu'elles pou-

\footnotetext{
* Comme prévu, I.B.A. (Industrial Biotechnology Association) vient de faire connaître sa position le 10 juin dernier: celte association demande que le N.I.H. ne cherche pas à breveter des séquences partielles de gènes ou des séquences de gènes dont la fonction biologique est inconnue, I. B.A. considère que ces séquences devraient être placées dans le domaine public. De plus, I.B.A. presse le gouvernement américain d'adopter unilatéralement de telles mesures pour tout ce qui concerne les agences fédérales américaines ou les activités financées en partie ou en totalité par cellesci. Enfin, celle association demande que le gouvernement des États-Unis cherche un accord international sur l'adoption de mesures similaires à celles énoncées ci-dessus. Celle prise de position constitue incontestablement un tournant dans le débat sur la brevetabilité des séquences d'ADN, et pourrait laisser présager un accord prochain entre toutes les parties et les pays intéressés.

Après I.B.A., c'est P.M.A. (Pharmaceutical Manufacturers Association) qui a demandé aux autorités américaines de renoncer aux brevets sur les
}

vaient être contre-incitatives au développement biotechnologique, forçant les compagnies à travailler sous licence avec le NIH. De plus, il souligna lui aussi le risque extraordinaire de conflits multiples d'enfreinte à la propriété intellectuclle créés par la possibilitć que les mêmes gènes soient couverts par des brevets sur des séquences partielles différentes.

Le porte-parole d'ABC (Association of Biotechnology Companies), la scconde association des entreprises de biotechnologie, regroupant plutôt des entreprises de taille moyenne, apporta son soutien à la politique du NIH, sans cependant faire référence à la nature particulière de cet objet brevetable que sont les séquences partielles d'ADN. Wolfgang Siebeck, du Consultative Group on International Agricultural Research, mit en évidence le danger de protection trop forte pour les pays en voie de développement, cmpêchant, par exemple, l'utilisation d'un produit spécifiquement utile à ces pays parce qu'il scrait protégé par un brevet concédé à des compagnies n'envisagcant pas de le développer. La dernic̀re séric d'interventions fut celle des représentants des pays de la CEE ct du responsable du burcau de la CEE à Washington. Pour la Grande-Bretagne, David Schapiro, du Nuffield Council on Bioethics et David Owen, du MRC, insistèrent sur la non-brevetabilité de séquences particlles d'ADN et appelèrent de leurs vœux un accord international pour reconnaître ce point. Le représentant du MRC indiqua que ce type de brevet risquait d'affaiblir la valeur et la force de ceux protégeant de réelles inventions en biotechnologie. La position française fut présentée par moimême pour ce qui concerne les séquences d'ADN et par Jacques Damagnez, du ministère des Affaires Étrangères, pour ce qui est de l'autre sujet abordé au cours de cet audit (mais non commenté ici) : la brevetabilité des variétés végétales et des animaux. Je développai l'idée que au-delà des arguments éthiques amenant à considérer comme choquante une appropriation intellectuelle de la séquence partielle ou totale des gènes humains, la démarche du NIH était parfaitement révolutionnaire en terme des rapports entre science et proprićté puisqu'il s'agissait ici de breveter (c'est-à-dire de demander une proprićté intellectuelle sur) une connaissance pure, et non pas un produit ou un procédé. Les séquences de Craig Venter sont en effet partielles, éventucllement non codantes, si bien que rien ne peut être dit de ce à quoi pourrait servir un produit dérivé du gène cn question, ni mĉme si ce gène a quelqu'intérêt physiologique ou pathologique. De plus, l'activité inventive nécessaire à l'obtention de ces bribes de sćquences est particulièrement faible, ni la synthèse d'ADNc ni le séquençage automatique sur machines Applied biosystem n'ćtant particulièrement originaux. Je fis également remarquer à Bcrnardine Healy que les brevets pris par des équipes françaises concernaient des gènes entiers de fonction connue et couvraicnt donc un procédé (diagnostic prénatal d'une maladic, fabrication d'un médicament ou d'un réactif), et ćventuellement un produit. Enfin, je notai qu'une dérive vers l'appropriation intellectuclle de la connaissance risquait de tarir la source à laquelle s'est alimentéc le progrès de nos socićtés industriclles depuis deux siècles, progrès nécessitant que l'inventeur puisse disposer librement d'une masse d'informations scientifiques, propriété communc du genre humain, grâce auxquelles il peut aboutir rapidement et dévclopper efficacement son invention. En conclusion, je plaidai donc pour un accord international destiné à préciser la place de l'" objet brevetable" dans le continuum qui va de l'accumulation des connaissances, grâce aux découvertes, jusqu'aux inventions et à leur développement économique. Le représentant de la Commission Europécnne à Washington, Gilbert Fayl, se fit l'écho de la recommandation unanime du EC Human Genome program's advisory body : celui-ci considère que les séquences partielles d'ADN ne sont pas brevetables et appelle de ses vœux, comme les délégués français et anglais, un accord international sur le sujet.

L'impression générale issue de cet audit est que, aux États-Unis, la thèse de la brevetabilité élargic à n'importe quel ćlément de connaissance particlle du génome humain est 
fortement soutenue par les autorités fédérales proches de la Présidence, mais continue de rencontrer d'importantes oppositions dans le milicu scientifique et dans des agences aussi importantes que le Department of Energy. L'attitude de l'industric biotechnologique est trc̀s intéressante. Si $\mathrm{ABC}$ s'est totalement ralliée aux thc̀ses du NIH, IBA, nettement plus importante puisque représentant $80 \%$ des investissements américains en biotechnologic, semble s'orienter vers une attitude plus réservée par rapport à ce type de brevets dont clle soupçonne qu'ils scront dissuasifs, dans nombre de cas, pour des développements ultérieurs.

L'opposition internationale est certaincment appelćc à jouer un rôle important dans l'issue de cette controverse. La position de la France a été particulièrement remarquéc, notre pays étant considéré comme ayant unc position de leader dans l'opposition à l'extension projetée du droit des brevets. C'est cela qui a justifić les vives attaques de la directrice du $\mathrm{NIH}$ contre la position française. En revanche, les forces, nombreuses et maintenant organisćes, qui continuent, aux États-Unis, à s'opposer à cette ćvolution se disent réellement encouragécs par les pressions interna- tionales, notamment celles venant de la France. Un autre ćlément expliquant la force de la position française cst la considération indéniable des scientifiques et des autorités américaines pour la qualité et l'importance du programme Génome français ; les Américains se disent particulic̀rement impressionnés par l'ensemble constitué du CEPH et du Généthon qui, pour bcaucoup d'entre eux, constituent une menace scientifique prise trc̀s au sérieux.

La position britannique est certainement affaiblie par les dépôts de demande de brevets du MRC aux États-Unis pour ses propres sćquences partielles : le NIH a remarqué que cette attitude, quel que soit le discours l'cntourant, correspondait au même souci que le sien : une position " conservatoire" en attendant que soit réglé le problème. A l'audit de Washington, cependant, les positions des intervenants britanniques furent, sans ambiguïté, hostiles à la brevetabilité de séquences partielles d'ADN sans fonction connue, même s'il est ćvident que le MRC joue ainsi sur les deux tableaux : l'obtention de ses brevets si telle doit être l'issuc, un certificat de conduite solidaire et rigoureux dans l'autre cas.

La position du représentant de la
Communauté Europécnne fut d'unc hostilité sans concessions aux brevets sur des ćléments intermédiaires de la recherche sur le génome, et fut ćcoutée avec une très grande attention par les Américains.

En conclusion, mon impression est qu'unc action concertée de la Communauté Europécnne pourrait avoir une influence importante sur l'évolution de la discussion en cours : d'une part, en renforçant la position et la détermination de ceux qui, aux ÉtatsUnis, s'opposent à l'extension projetée du droit des brevets, d'autre part, dans lc cas, qui est loin d'ĉtre impossible, où le Bureau Amćricain des Brevets donnerait partiellement satisfaction au NIH, en mettant l'Europe (et peut-être le Japon) en bonne position dans les négociations internationales futures sur ce sujet

\section{Axel Kahn}

ICGM, Inserm U. 129, 24, rue du Faubourg-Saint-Jacques, 75014 Paris, France.

\section{TIRÉS A PART}

A. Kahn

En date du 20 juin et de source bien informée, il semble que le NIH envisage maintenant d'harmoniser sa position avec celles du DOE, de la France et de l'Angleterre. 